\title{
GUARANTEED PERFORMANCE CONTROL FOR DISCRETE-TIME LQG PROBLEMS
}

\author{
Duan $\mathbf{L i}^{*, 1}$ Peilin Fu ${ }^{*}$ Fucai Qian ${ }^{* *}$
}

\author{
* Department of Systems Engineering and Engineering \\ Management, The Chinese University of Hong Kong \\ ** School of Automation and Information Engineering, \\ Xi'an University of Technology
}

\begin{abstract}
Guaranteed performance control is considered in this paper for discretetime LQG problems by minimizing the probability that the performance index is over a preselected threshold. It is proven that this guaranteed performance control problem can be converted into a mean-variance control problem which can be solved by using an embedding scheme. An optimal open-loop feedback control law is derived for the guaranteed performance control of discrete-time LQG problems. Copyright (C)2005 IFAC
\end{abstract}

Keywords: Guaranteed performance control, linear-quadratic Gaussian control, Stochastic control, Dynamic programming, Mean-variance control

\section{INTRODUCTION}

Without a doubt, linear-quadratic control represents one of the most prominent successes in control theory, largely due to its wide applications and its mathematical elegance in tractability. We consider in this paper the following discrete-time linear-quadratic Gaussian optimal control problem:

$$
\begin{gathered}
(M) \quad \min E\{J\}=E\left\{x^{T}(N) Q(N) x(N)\right. \\
\left.+\sum_{k=0}^{N-1}\left[x^{T}(k) Q(k) x(k)+u^{T}(k) R(k) u(k)\right]\right\} \\
\text { s.t. } x(k+1)=A x(k)+B u(k)+\xi(k)
\end{gathered}
$$

where $x(k) \in R^{n}$ is the state, $u(k) \in R^{m}$ is the control, $A$ and $B$ are matrices of appropriate dimensions, $\{\xi(k)\}$ is a sequence of white Gaussian random noises with $\xi(k) \sim N\left(0, \Theta_{\xi}\right)$ and

\footnotetext{
1 This research was partially supported by the Research Grants Council of Hong Kong under Grants CUHK 4180/03E and CUHK 4245/04E.
}

the initial state $x(0)$ is known. Furthermore it is assumed that for all $k$ the matrices $Q(k)$ and $R(k)$ are symmetric positive-semidefinite and positivedefinite, respectively.

The optimal control to the above linear-quadratic Gaussian control problem is well known as

$$
u^{*}(k)=\mu_{k}^{*}(x(k))=L(k) x(k)
$$

where

$$
\begin{aligned}
L(k)= & -\left[B^{T} S(k+1) B+R(k)\right]^{-1} B^{T} S(k+1) A \\
S(k)= & A^{T}\left[S(k+1)-S(k+1) B\left(B^{T} S(k+1) B\right.\right. \\
& \left.+R(k))^{-1} B^{T} S(k+1)\right] A+Q(k)
\end{aligned}
$$

with boundary condition of $S(N)=Q(N)$. The corresponding optimal expected performance index is

$$
\begin{aligned}
\left.E\{J\}\right|_{\left\{u^{*}(k)\right\}} & =x(0)^{T} S(0) x(0) \\
& +\sum_{k=0}^{N-1} E\left\{\xi^{T}(k) S(k+1) \xi(k)\right\} .
\end{aligned}
$$


The traditional linear-quadratic optimal stochastic control theory only concerns a sole objective of minimizing the expected value of the performance index. Each realization of the optimal control policy, however, can only result in a single value of the performance index $J$. Figure 1 depicts the distribution of the values of the performance index from 1000 simulation runs for the example problem considered in Section 4 of this paper. Although the optimal expected performance index is 49.45 , the distribution of the realized values of $J$ ranges widely from 25 to 120 . In many situations, the optimal control policy is only applied to the process once. It is thus evident that a control over worst-case scenarios is necessary.

In investment, one common goal of investors is to minimize the probability that their terminal wealth is below a preselected threshold which may be viewed as a "disaster" level ( $\mathrm{Li}$ et al., 1998). Finding an optimal policy in order to avoid trapping into the domain of this defined "disaster" is the principle of safety-first proposed in portfolio selection by Roy (Roy, 1952). Motivated by the principle of safety-first, we will explore in this paper how to achieve a guaranteed performance for discrete-time LQG problems. More specifically, the guaranteed performance control problem is to minimize the probability that the performance index is over a preselected threshold.

Let $\delta$ be the preselected threshold. The optimal control problem for guaranteed performance is formulated as follows,

$$
\begin{aligned}
& (G P) \quad \min P(J \geq \delta) \\
& \text { s.t. } x(k+1)=A x(k)+B u(k)+\xi(k)
\end{aligned}
$$

where $J$ is defined in (1) and the value of $\delta$ is assumed to be greater than $E(J)$ associated with the control policy adopted. Define by $I^{k}$ the information set at time $k$, namely,

$$
\begin{aligned}
I^{k}=\{ & x(0), u(0), x(1), u(1), \\
& \ldots, x(k-1), u(k-1), x(k)\} .
\end{aligned}
$$

A control is admissible if it is nonanticipated, i.e., $u(k)$ only depends on $I^{k}$. Of course, the search of optimal control for $(G P)$ is confined within the class of admissible controls.

\section{RELATIONSHIP WITH MEAN-VARIANCE CONTROL}

In this section, we will examine the relationship between problem $(G P)$ and the mean-variance control problem studied in (Li et al., 2003).

From Bienayme-Tchebycheff inequality, we have

$$
\begin{aligned}
P(J \geq \delta) & =P(J-E(J) \geq \delta-E(J)) \\
& \leq \frac{\operatorname{Var}(J)}{(E(J)-\delta)^{2}} .
\end{aligned}
$$

Minimizing $P(J \geq \delta)$ can be achieved by minimizing its upper bound. Therefore, we consider the following surrogate problem of $(G P)$,

$$
\begin{aligned}
&(S G P) \quad \min U\{E(J), \operatorname{Var}(J)\} \\
&= \frac{\operatorname{Var}(J)}{(E(J)-\delta)^{2}} \\
& \text { s.t. } x(k+1)=A x(k)+B u(k)+\xi(k) .
\end{aligned}
$$

A control $u^{*}$ is called an efficient control if there exists no other admissible $u$ such that

$$
\begin{aligned}
\left.E(J)\right|_{u} & \leq\left. E(J)\right|_{u^{*}} \\
\left.\operatorname{Var}(J)\right|_{u} & \leq\left.\operatorname{Var}(J)\right|_{u^{*}}
\end{aligned}
$$

with at least one strict inequality.

Lemma If $u^{*}$ is an optimal control of problem $(S G P)$, then $u^{*}$ must be an efficient control.

Proof: If $u^{*}$ is not an efficient control, then there exists an admissible $u$ such that

$$
\begin{gathered}
\left.E(J)\right|_{u} \leq\left. E(J)\right|_{u^{*}} \\
\left.\operatorname{Var}(J)\right|_{u} \leq\left.\operatorname{Var}(J)\right|_{u^{*}}
\end{gathered}
$$

with at least one strict inequality. Since $U$ is a strictly increasing function of both $E(J)$ and $\operatorname{Var}(J)$ under the assumption of $E(J) \leq \delta$, we have

$$
\left.U\{E(J), \operatorname{Var}(J)\}\right|_{u}<\left.U\{E(J), \operatorname{Var}(J)\}\right|_{u^{*}}
$$

which is a contradiction to the assumption that $u^{*}$ is an optimal control of problem $(S G P)$.

Problem $(S G P)$ is difficult to solve and an analytical solution is impossible to obtain. Under a convexity assumption, all efficient controls, including the optimal control of problem $(S G P)$, can be generated by the following tractable auxiliary problem,

$$
\begin{aligned}
& (A(\tilde{\mu})) \quad \min \operatorname{Var}(J)+\tilde{\mu} E(J) \\
& \text { s.t. } x(k+1)=A x(k)+B u(k)+\xi(k)
\end{aligned}
$$

where $\tilde{\mu}$ is a positive parameter.

Denote by $U_{E}$ and $U_{V}$ the partial derivatives of $U$ with respect to $E(J)$ and $\operatorname{Var}(J)$, respectively,

$$
\begin{aligned}
& U_{E}=\frac{\partial U}{\partial E(J)}=-2 \frac{\operatorname{Var}(J)}{(E(J)-\delta)^{3}}>0 \\
& U_{V}=\frac{\partial U}{\partial \operatorname{Var}(J)}=\frac{1}{(E(J)-\delta)^{2}}>0 .
\end{aligned}
$$

where the assumption that $\delta$ is greater than $E(J)$ is used. 
Theorem Suppose that $u^{*}$ is an optimal control of $\left(A\left(\tilde{\mu}^{*}\right)\right)$ with $\tilde{\mu}^{*}>0$. A necessary condition that $u^{*}$ is also an optimal control of the problem $(S G P)$ is

$$
\tilde{\mu}^{*}=\left.\frac{U_{E}}{U_{V}}\right|_{u^{*}}=-\left.2 \frac{\operatorname{Var}(J)}{E(J)-\delta}\right|_{u^{*}}
$$

Proof: Under the convexity condition, the efficient frontier in the $\{E(J), \operatorname{Var}(J)\}$ space can be parameterized by the coefficient $\tilde{\mu}$. In other words, every point on the efficient frontier can be represented by $\{E[J(\tilde{\mu})], \operatorname{Var}[J(\tilde{\mu})]\}$. Therefore problem $(S G P)$ can be reduced in abstract to the following equivalent form,

$$
\min _{\tilde{\mu} \geq 0} U\{E[J(\tilde{\mu})], \operatorname{Var}[J(\tilde{\mu})]\}
$$

If optimal $\tilde{\mu}^{*}>0$, the first-order necessary condition is

$$
\left.U_{E}\right|_{u^{*}} \frac{\partial E\left[J\left(\tilde{\mu}^{*}\right)\right]}{\partial \tilde{\mu}}+\left.U_{V}\right|_{u^{*}} \frac{\partial \operatorname{Var}\left[J\left(\tilde{\mu}^{*}\right)\right]}{\partial \tilde{\mu}}=0
$$

On the other hand, when $u^{*}$ solves problem $\left(A\left(\tilde{\mu}^{*}\right)\right)$, we have the following from (Reid and Citron, 1971),

$$
\tilde{\mu} \frac{\partial E\left[J\left(\tilde{\mu}^{*}\right)\right]}{\partial \tilde{\mu}}+\frac{\partial \operatorname{Var}\left[J\left(\tilde{\mu}^{*}\right)\right]}{\partial \tilde{\mu}}=0 .
$$

Vector $\left[U_{E}\left(u^{*}\right), U_{V}\left(u^{*}\right)\right]$ is proportional to $\left[\tilde{\mu}^{*}, 1\right]$. Thus, we must have $\tilde{\mu}^{*}=U_{E} /\left.U_{V}\right|_{u^{*}}$.

A key finding is that if we can find the optimal $\tilde{\mu}^{*}$ that satisfies optimal condition (2), then the optimal control to problem $\left(A\left(\tilde{\mu}^{*}\right)\right)$ is a candidate for the optimal control of problem $(S G P)$.

\section{OPTIMAL OPEN-LOOP FEEDBACK SOLUTION}

The auxiliary problem $(A(\tilde{\mu}))$ is a mean-variance control problem. Such kind of problems was first investigated by Sain and Liberty (Sain, 1966) (Sain and Liberty, 1971) for continuous-time linear-quadratic Gaussian systems. Li, Qian and $\mathrm{Fu}$ (Li et al., 2003) investigated the mean-variance control problem for discrete-time LQG problem with the following formulation for a given nonzero $\mu \in(0,1]$,

$$
\begin{aligned}
& (A(\mu)) \quad \min \mu E(J)+(1-\mu) \operatorname{Var}(J) \\
& \text { s.t. } x(k+1)=A x(k)+B u(k)+\xi(k) .
\end{aligned}
$$

Parameter $\mu$ represents a trade-off between two conflicting objectives, minimizing the expected performance index and minimizing the variance. By using an embedding scheme to overcome the non-separability in variance minimization, an analytical optimal open-loop feedback control law is obtained for $(A(\mu))$ as follows,

$$
u^{*}(k)=-\Gamma(k) x(k)
$$

where

$$
\begin{aligned}
& \Gamma(k)=\Gamma_{1}(k)+\Gamma_{2}(k) \psi_{k+1}^{k-N} M(k) \\
& \Gamma_{1}(k)=D^{-1}(k) B^{T} S(k+1) A \\
& \Gamma_{2}(k)=\frac{1-\mu}{\mu} D^{-1}(k) B^{T} \\
& D(k)=B^{T} S(k+1) B+R(k) \\
& S(k)=A^{T} S(k+1) A-\Gamma_{1}^{T}(k) D(k) \Gamma_{1}(k) \\
& +Q(k) \\
& M(k)=4 \Omega^{-1}(k) H(k) C(k) \\
& \Omega(k)=I+\frac{4(1-\mu)}{\mu} H(k) \Psi^{T}(k) \Lambda(k) \Psi(k) \\
& C(k)=\left[\Phi^{T}(k, k), \Phi^{T}(k, k+1)\right. \\
& \left., \ldots, \Phi^{T}(k, N-1)\right]^{T} \\
& H(k)=\operatorname{diag}(Q(k+1), \cdots, Q(N)) \\
& \times\left(\begin{array}{ccc}
W(k+1, k+1) & \cdots & W(k+1, N) \\
\vdots & \vdots & \vdots \\
W(N, k+1) & \cdots & W(N, N)
\end{array}\right) \\
& \times \operatorname{diag}(Q(k+1), \cdots, Q(N)) \text {, } \\
& \Psi(k)=\left[\begin{array}{c}
\psi_{k+1}^{k-N} \\
\vdots \\
\psi_{N}^{k-N}
\end{array}\right] \\
& =\left[\begin{array}{cccc}
I & \Phi^{T}(k+1, k+1) & \cdots & \Phi^{T}(k+1, N-1) \\
0 & I & \cdots & \Phi^{T}(k+2, N-1) \\
\vdots & \vdots & \vdots & \vdots \\
0 & 0 & \cdots & I
\end{array}\right] \\
& \Phi\left(k_{0}, k\right)=\left(A-B \Gamma_{1}(k)\right)\left(A-B \Gamma_{1}(k-1)\right) \cdots \\
& \times\left(A-B \Gamma_{1}\left(k_{0}\right)\right), k \geq k_{0} \\
& \Lambda(k)=\operatorname{Diag}\left(B D^{-1}(k) B^{T}, \ldots,\right. \\
& \left.B D^{-1}(N-1) B^{T}\right) \\
& W(k, j)=\sum_{i=1}^{\min \{k, j\}} A^{k-i} \Theta_{\xi}\left(A^{j-i}\right)^{T}
\end{aligned}
$$

with boundary condition $S(N)=Q(N)$.

If we let

$$
\tilde{\mu}=\frac{\mu}{1-\mu},
$$

problem $(A(\tilde{\mu}))$ is equivalent to $(A(\mu))$. The optimality condition in (2) can be now expressed in terms of $\mu$,

$$
\frac{\mu}{1-\mu}=-2 \frac{\operatorname{Var}(J)}{E(J)-\delta}
$$

namely

$$
\mu=\frac{2 \operatorname{Var}(J)}{2 \operatorname{Var}(J)-E(J)+\delta} .
$$

Notice that both $E(J)$ and $\operatorname{Var}(J)$ are $\mu$ dependent. 
In order to check the optimal condition (4) for parameter $\mu, E(J)$ and $\operatorname{Var}(J)$ need to be calculated for given $\mu$. More specifically, we need to calculate $E(J)$ and $\operatorname{Var}(J)$ after applying the optimal control law in (3). Notice that the term $M(k)$ in the optimal control law is $\mu$ dependent. In the following, an approach using moment-generating function will be derived for calculating $E(J)$ and $\operatorname{Var}(J)$ for given $\mu$.

Define $\rho(\theta, k, x)$ as the moment-generating function of the cost-to-go at stage $k$,

$$
\rho(\theta, k, x)=E\left\{e^{\theta J(k, x)}\right\},
$$

where

$$
\begin{aligned}
J(k, x) & =x^{\prime}(N) Q(N) x(N) \\
& +\sum_{t=k}^{N-1}\left[x^{\prime}(t) Q(t) x(t)+u^{\prime}(t) R(t) u(t)\right]
\end{aligned}
$$

with $x(k)=x$.

For fixed $\theta$, the moment-generating function has been proved in $(\mathrm{Fu}, 2003)$ as follows when applying the optimal control law in (3),

$$
\rho(\theta, k, x)=\prod_{t=k}^{N-1} \sigma(t) \exp \left\{\theta\left[x^{\prime}(k) V(k) x(k)\right]\right\}
$$

where

$$
\begin{aligned}
& \sigma(t)= \sqrt{\frac{\left|\left[\Theta_{\xi}^{-1}-2 \theta V(t+1)\right]^{-1}\right|}{\left|\Theta_{\xi}\right|}}, \\
& t=N-1, N-2, \ldots, k \\
& V(k)=Q(k)+\Gamma^{\prime}(k) R(k) \Gamma(k) \\
&+[A-B \Gamma(k)]^{\prime} \tilde{V}(k+1)[A-B \Gamma(k)] \\
& \tilde{V}(k)=V(k)+2 \theta V^{\prime}(k)\left[\Theta_{\xi}^{-1}-2 \theta V(k)\right]^{-1} \\
& \quad \times V(k)
\end{aligned}
$$

with boundary condition $V(N)=Q(N)$.

The mean and variance of the performance index can be obtained from the moment-generating function as follows:

$$
\begin{aligned}
E(J) & =\left.\frac{\partial \rho(\theta, 0, x(0))}{\partial \theta}\right|_{\theta=0}, \\
\operatorname{Var}(J) & =\left.\frac{\partial^{2} \rho(\theta, 0, x(0))}{\partial \theta^{2}}\right|_{\theta=0}-E^{2}(J) .
\end{aligned}
$$

Numerical algorithm can be devised to find out such $\mu$ s that satisfy the optimal necessary condition (4). The corresponding feedback optimal control law $u^{*}(k)$ specified in equation (3) solves the guaranteed performance problem.

\section{IMPLEMENTATION OF THE CONTROL LAW AND ILLUSTRATIVE EXAMPLE}

The above derived optimal feedback control law for the guaranteed performance control problem $(S G P)$ can be implemented using the following algorithm.

\section{Optimal Control Algorithm for $(S G P)$ :}

Step 0 Choose a very small positive number $\epsilon$ for the error tolerance. Calculate noise matrix $W(i, j)$ for all $i, j=1,2, \ldots, N-1$. Select $\mu_{1}>0$ such that $\mu_{1}$ is very close to 0 and select $0<\mu_{2}<1$ such that $\mu_{2}$ is very close to 1 . For each $\mu_{1}$ and $\mu_{2}$, perform the following inner loop.

Begin of the inner loop for solving $(A(\mu))$ with a given parameter $\mu$ :

Step 1: Set $k=0$.

Step 2: For given $\mu$, calculate $\left\{\Gamma_{1}(k)\right\},\left\{\Gamma_{2}(k)\right\}$, $\overline{\{D(k)}\},\{S(k)\}$.

Step 3: Calculate the $\{\Psi(k)\},\{\Lambda(k)\},\{C(k)\}$, $\overline{\text { and }\{M}(k)\}$.

Step 4: Calculate the $\{\Gamma(k)\}$.

Step 5: Observe $x(k)$ and calculate the on-line $\overline{\text { control }} u(k)=-\Gamma(k) x(k)$.

Step 6: Apply $u(k)$ to the system. If $k=N-1$, go to next step; otherwise, set $k=k+1$, go back to Step 2.

Step 7: Calculate $E(J)$ and $(\operatorname{Var}(J))$.

End of the inner loop

Step 8: Define $\tau=\mu-\frac{2 \operatorname{Var}(J)}{2 \operatorname{Var}(J)-E(J)+\delta}$. If $|\tau| \leq$ $\bar{\varepsilon}$, the control corresponding to $\mu$ is optimal for $(S G P)$. Otherwise, do line search to select a new value of $\mu$ and go back to Step 1 .

Example: Consider the following scalar LQG problem:

$$
\begin{array}{ll} 
& \min E(J) \\
\text { s.t. } & x(k+1)=0.8 x(k)+0.5 u(k)+\xi(k), \\
& k=0,1, \ldots, 19, x(0)=4
\end{array}
$$

where the performance index is

$$
J=x^{2}(20)+\sum_{k=0}^{k=19}\left[x^{2}(k)+u^{2}(k)\right] .
$$

The system noises are white Gaussian with $\xi(k) \sim$ $N(0,0.6)$.

Applying the conventional LQG optimal control, the distribution of the performance index is demonstrated in Figure 1 for 1000 runs. It is evident that the dispersion of the performance index is wide. Note that the corresponding mean and variance associated with the LQG optimal control 


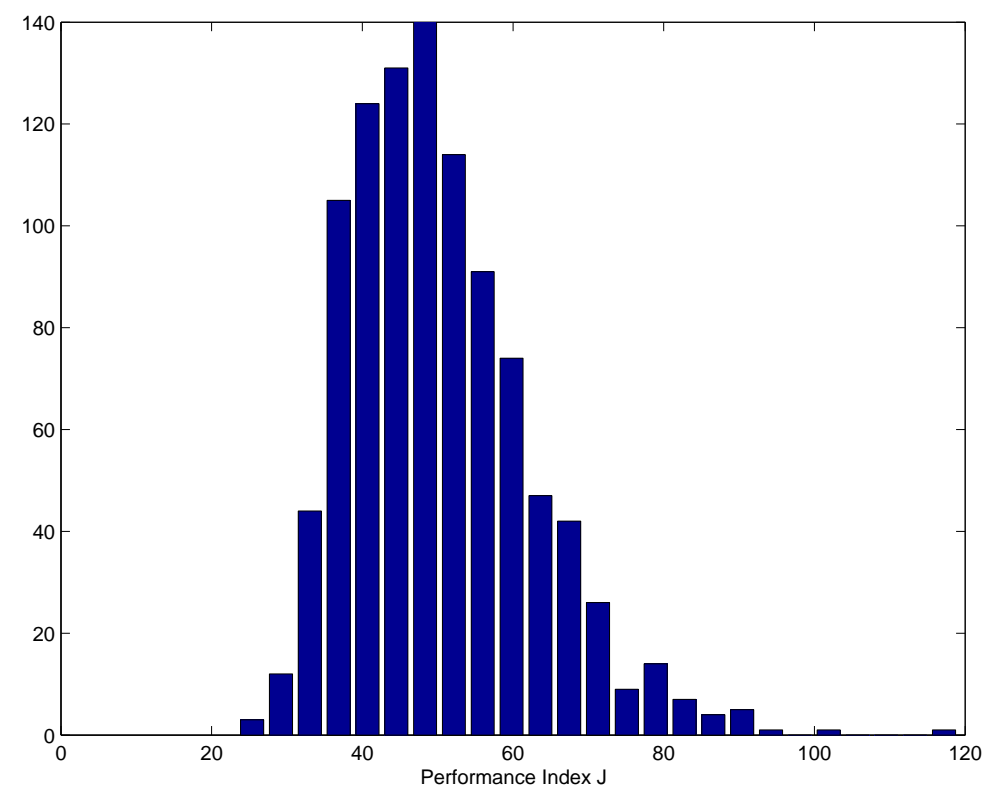

Fig. 1. Distribution of performance index of the Example Problem (Using the conventional LQG optimal control)

are $E(J)=49.45342$ and $\operatorname{Var}(J)=133.8272$, respectively.

If we would like to control the probability that the performance index is greater than a threshold 80 , the following guaranteed performance control problem can be formulated,

$$
\begin{gathered}
\min P(J \geq 80) \\
\text { s.t. } x(k+1)=0.8 x(k)+0.5 u(k)+\xi(k), \\
k=0,1, \ldots, 19, x(0)=4 .
\end{gathered}
$$

For each given $\mu$ we convert the above problem to problem $(A(\mu))$ which can be solved by using the optimal feedback control law (3). Applying the control law to the system enables a calculation of the mean and variance of the performance index. When the optimality condition (4) is not satisfied for the given $\mu$, a bisection search is used to search for a better $\mu$ in $(0,1)$. The algorithm terminates with $\mu^{*}=0.88869$ that satisfies (4) with corresponding $E(J)=50.01$ and $\operatorname{Var}(J)=117.65$. Substituting these values into the Bienaym-Tchebycheff inequality (1) reveals that the probability that the performance index $J$ is larger than 80 will be controlled under 0.1308 . Applying the guaranteed performance control for $\delta=80$, the distribution of the performance index is given in Figure 2 for 1000 runs. It is evident that the dispersion of the performance index is much improved under the guaranteed performance control.

Table 1 further demonstrates results of the guaranteed performance control for various $\delta$. For each value of $\delta$, the optimal value of $\mu$ is calculated which is listed in the second column. One hundred
Monte Carlo simulation runs were performed for each value of $\delta$. For 100 samples, the number that the performance index exceeds $\delta$ was recorded for the guaranteed performance control and the conventional LQG optimal control, respectively. The two numbers of outliers above $\delta$ under the guaranteed performance control and the conventional LQG optimal control are denoted by $O L_{\delta}^{G}$ and $O L_{\delta}^{C}$, respectively. In the second part of the table, under the guaranteed performance control for each $\delta, E(J)$ and $V(J)$ denote the derived theoretical expected value and the variance of the performance index $J$, respectively, $\tilde{E}(J)$ and $\tilde{V}(J)$ denote the sample expected value and sample variance of the performance index $J$, respectively, and $\operatorname{Pr}_{\delta}=\frac{\operatorname{Var}(J)}{(E(J)-\delta)^{2}}$ is the theoretical value of the upper bounded of the probability that $J \geq \delta$.

TABLE 1. Simulation Results of Guaranteed Performance Control

\begin{tabular}{cccc}
\hline$\delta$ & $\mu$ & $O L_{\delta}^{G}$ & $O L_{\delta}^{C}$ \\
110 & 0.79 & 0 & 1 \\
100 & 0.82 & 0 & 3 \\
95 & 0.84 & 0 & 6 \\
90 & 0.85 & 1 & 8 \\
85 & 0.87 & 3 & 14 \\
80 & 0.89 & 5 & 19 \\
75 & 0.90 & 20 & 36 \\
\hline
\end{tabular}

\begin{tabular}{ccccccc}
\hline$\delta$ & $\mu$ & $E(J)$ & $\tilde{E}(J)$ & $V(J)$ & $\tilde{V}(J)$ & $\operatorname{Pr}_{\delta}$ \\
110 & 0.79 & 50.77 & 51.53 & 110.64 & 110.23 & $3.15 \%$ \\
100 & 0.82 & 50.51 & 50.43 & 112.59 & 102.30 & $4.60 \%$ \\
95 & 0.84 & 50.38 & 50.15 & 113.69 & 114.07 & $5.71 \%$ \\
90 & 0.85 & 50.26 & 49.52 & 114.88 & 106.54 & $7.27 \%$ \\
85 & 0.87 & 50.13 & 49.75 & 116.20 & 109.04 & $9.56 \%$ \\
80 & 0.89 & 50.01 & 49.99 & 117.65 & 118.16 & $13.08 \%$ \\
75 & 0.90 & 49.89 & 50.01 & 119.28 & 127.33 & $18.91 \%$ \\
\hline
\end{tabular}

It is obvious from the table that the number of outliers above $\delta$ under the guaranteed per- 


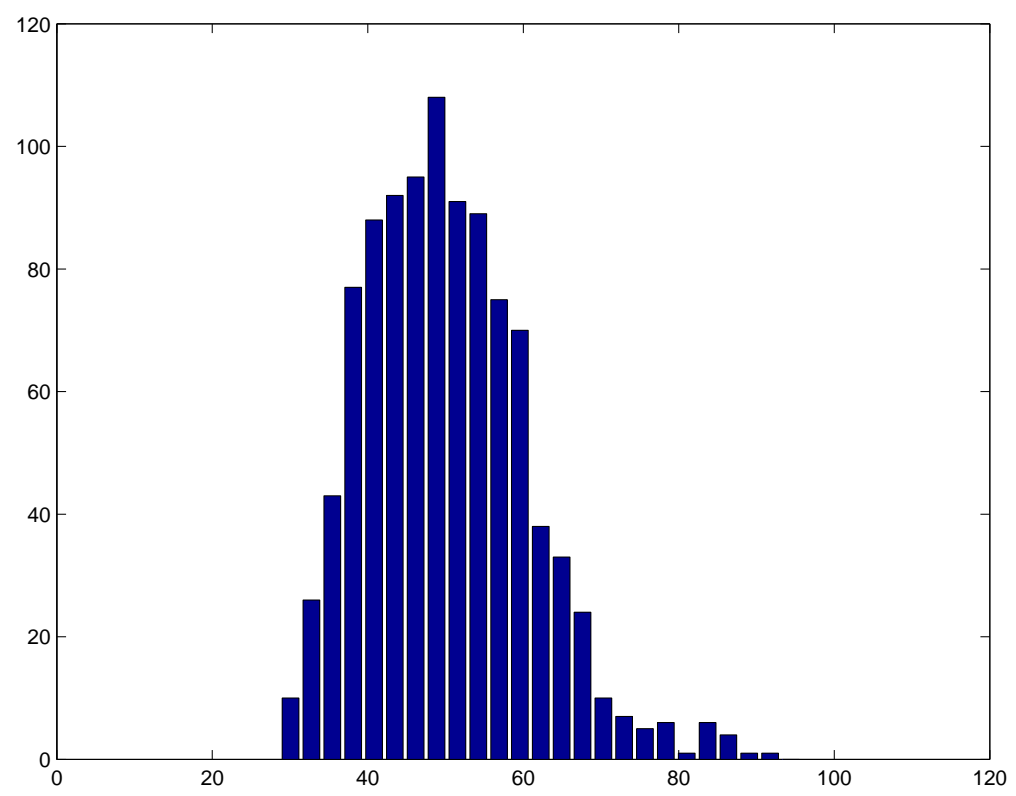

Fig. 2. Distribution of performance index of the Example Problem (Using the guaranteed performance control for $\delta=80$ )

formance control is much less than the number of outliers above $\delta$ under the conventional LQG control. Comparing the value of $\frac{O L_{\delta}^{G}}{100}$ with the upper bound derived based on the BienaymeTchebycheff inequality for each $\delta$, we can conclude that the upper bound is quite loose.

\section{CONCLUSION}

In the traditional discrete-time LQG control problem, only the expected value of the performance index is under control. In applications, it is often the case that the dispersion of the performance index spans widely which is not desirable in many situations. In this paper, a kind of guaranteed performance control problem is investigated. The derived optimal open-loop feedback control law can be used to minimize the probability that the performance index is over a preselected threshold which may be viewed as a "disaster" level.

\section{REFERENCES}

Fu, P. L. (2003). Variance Minimization and Dual Control. PhD thesis. Department of Systems Engineering and Engineering Management, The Chinese University of Hong Kong.

Li, D., F. C. Qian and P. L. Fu (2003). Meanvariance control for discrete-time LQG problems. In: Preceedings of the 2003 American Control Conference. pp. 4444-4449.

Li, D., T. F. Chan and W. L. Ng (1998). Safetyfirst dynamic portfolio selection. Dynamics of Continuous, Discrete and Impulsive Systems 4(4), 585-600.
Reid, R. W. and S. J. Citron (1971). On noninferior performance index vector. Journal of Optimization Theory and Application 7, 1128.

Roy, A. D. (1952). Safety first and the holding assets. Econometrica 20, 431-449.

Sain, M. K. (1966). Control of linear systems according to the minimal variance criterion: A new approach to the disturbance problem. IEEE Transactions on Automatic Control AC-11(1), 118-122.

Sain, M. K. and S. R. Liberty (1971). Performance measure densities for a class of lqg control systems. IEEE Transactions on Automatic Control AC-16(5), 431-439. 